\title{
Protection of immunized mice and swine to challenge exposure with Erysipelothrix rhusiopathiae strains obtained from recent swine erysipelas outbreaks in Japan
}

\author{
Ho To, Nobuyuki Tsutsumi, Akihiro Tazumi, Takashi Kamada, Tetsuji Nagano, Shinya \\ Nagai, Akira Iwata, Tetsuo Nunoya \\ Nippon Institute for Biological Science, Shinmachi, Ome, Tokyo, Japan \\ Received August 16, 2012 \\ Accepted February 19, 2013
}

\begin{abstract}
The aim of the study was to investigate the protective effect provided by immunization with commercial erysipelas vaccines in mice and conventional pigs to challenge with the field strains (serotype 1a) of Erysipelothrix rhusiopathiae. Sequence analysis of surface protective antigen (spaA) gene revealed that the strains were different from those reported previously. A total of 150 mice immunized subcutaneously with live or inactivated vaccines were protected against challenge exposure to one reference and four field strains. Seven conventional pigs immunized intramuscularly with inactivated vaccines developed specific antibodies against the SpaA protein of E. rhusiopathiae and were protected against challenge with two field strains. Our study is the first to demonstrate that the commercially available vaccines could protect animals against challenge with the most recently isolated SpaA-type strains of E. rhusiopathiae.
\end{abstract}

Inactivated vaccine, live vaccine, recombinant SpaA protein, ELISA, anti-SpaA antibody

Live and inactivated erysipelas vaccines prepared from Erysipelothrix rhusiopathiae (E. rhusiopathiae) of serotypes 1a and 2, respectively, have been shown to elicit crossprotective immune responses in immunized mice and pigs against challenge with strains of serotypes 1 and 2 (Wood et al. 1981; Sawada and Takahashi 1987).

In Japan, a live vaccine prepared from the acriflavine-fast attenuated strain Koganei 65-0.15 of serotype 1a has been used for a long time (Sawada and Takahashi 1987). Additionally, three kinds of inactivated vaccines, a formalin-inactivated aluminumadsorbed vaccine prepared from Tama-96 strain (serotype 2) and two kinds of $\mathrm{NaOH}-$ treated oil adjuvant vaccines, formulated with Kyoto strain (serotype 2) or M2 strain (serotype 2), have been available commercially since the end of 1990s (Imada et al. 2003).

Recently, several studies have focused on Spa proteins of E. rhusiopathiae responsible for eliciting highly protective antibodies (Imada et al. 1999; Ingebritson et al. 2010). The Spa proteins can be classified into three molecular species, named SpaA (produced by serotypes 1a, 1b, 2, 5, 8, 9, 12, 15, 16, 17, and N), SpaB (produced by serotypes 4, 6, 11, 19, and 21) and SpaC (produced by serotype 18) (To et al. 2010).

During 2008-2011 many outbreaks of erysipelas have been reported among vaccinated and non-vaccinated swine in Japan. Nucleotide and amino acid sequence analysis of a 432-bp hypervariable region in spaA gene, involved in immunoprotection of SpaA, of the 83 recent field strains revealed that these strains were different from those reported previously (Nag a i et al. 2008), and could be divided into 3 groups: group 1 (3 strains of serotype 1a) had Ala-195 and Ile-203; group 2 (76 strains of serotype 1, and 3 of untypeable) had Asp-195 and Met-203; and group 3 (one strain of serotype 2a) had Asn-195 and Ile-203 (To et al. 2012).

The experiments described in the present study were undertaken to determine whether immunity induced in mice and conventional pigs with the live or inactivated vaccines would withstand challenge exposure with E. rhusiopathiae strains obtained from recent swine erysipelas (SE) outbreaks in Japan. 


\section{Materials and Methods}

Bacterial strains and growth conditions

Characteristics of E. rhusiopathiae strains used in the study are listed in Table 1. The bacteria were grown in tryptose phosphate broth supplemented with $1 \%$ proteose peptone no. 3 (BD diagnostic systems, Detroit, Michigan) and $0.1 \%$ Tween $80(\mathrm{pH} 7.8)$.

Table 1. Some characteristics of Erysipelothrix rhusiopathiae strains used in the study

\begin{tabular}{|c|c|c|c|c|c|c|c|}
\hline \multirow[b]{2}{*}{ Strain (group) (serotype) } & \multirow[b]{2}{*}{ Prefecture } & \multirow[b]{2}{*}{$\begin{array}{c}\text { Relevant } \\
\text { characteristic }\end{array}$} & \multirow{2}{*}{$\begin{array}{l}\text { History of } \\
\text { erysipelas } \\
\text { vaccination }\end{array}$} & \multicolumn{4}{|c|}{ Substitutions in nucleotide (amino acid position) in the spa $\mathrm{A}$ gen } \\
\hline & & & & $\begin{array}{c}\text { Nucleotide } \\
\text { (aa 185) }\end{array}$ & $\begin{array}{c}\text { Nucleotide } \\
\text { (aa 195) }\end{array}$ & $\begin{array}{c}\text { Nucleotide } \\
\text { (aa 203) }\end{array}$ & $\begin{array}{c}\text { Nucleotide } \\
\text { (aa 257) }\end{array}$ \\
\hline Koganei 65-0.15 (1a) & & $\begin{array}{c}\text { Japanese official } \\
\text { live vaccine strain }\end{array}$ & & $\begin{array}{c}\mathrm{CCC} \\
\text { (proline) }\end{array}$ & $\begin{array}{c}\text { GAT } \\
\text { (aspartic acid) }\end{array}$ & $\begin{array}{c}\text { ATT } \\
\text { (isoleucine) }\end{array}$ & $\begin{array}{c}\text { ATT } \\
\text { (isoleucine) }\end{array}$ \\
\hline Fujisawa (1a) & & $\begin{array}{l}\text { Japanese official } \\
\text { challenge strain }\end{array}$ & & $\begin{array}{c}\mathrm{CCC} \\
\text { (proline) }\end{array}$ & $\begin{array}{c}\text { GAT } \\
\text { (aspartic acid) }\end{array}$ & $\begin{array}{c}\text { ATT } \\
\text { (isoleucine) }\end{array}$ & $\begin{array}{c}\text { CTT } \\
\text { (leucine) }\end{array}$ \\
\hline Tama-96 (2) & & $\begin{array}{l}\text { Inactivated } \\
\text { vaccine strain }\end{array}$ & & $\begin{array}{c}\text { CCA } \\
\text { (proline) }\end{array}$ & $\begin{array}{c}\text { GAT } \\
\text { (aspartic acid) }\end{array}$ & $\begin{array}{c}\text { ATT } \\
\text { (isoleucine) }\end{array}$ & $\begin{array}{c}\underline{\text { CTT }} \\
\text { (leucine) }\end{array}$ \\
\hline Kumamoto-S1 (1) (1a) & Kumamoto & Septicaemia & Live vaccine & $\begin{array}{c}\mathrm{CCC} \\
\text { (proline) }\end{array}$ & $\begin{array}{c}\mathrm{GCT} \\
\text { (alanine) }\end{array}$ & $\begin{array}{c}\text { ATT } \\
\text { (isoleucine) }\end{array}$ & $\begin{array}{c}\text { ATT } \\
\text { (isoleucine) }\end{array}$ \\
\hline Gunma-657 (2) (1a) & Gunma & Septicaemia & Inactivated vac. & & & & \\
\hline Gunma-649 (2) (1a) & Gunma & Septicaemia & Inactivated vac. & $\mathrm{CCC}$ & GAT & AT $\underline{G}$ & ATT \\
\hline Nagano-16 (2) (1a) & Nagano & Septicaemia & Nonvaccination & (proline) & (aspartic acid) & (methionine) & (isoleucine) \\
\hline
\end{tabular}

${ }^{1}$ History of erysipelas vaccination of pig herd from which the strain was isolated

\section{Vaccines}

The erysipelas vaccines (Nisseiken Co., Ome, Japan) used in the study were "Nisseiken" Swine erysipelas inactivated vaccine (SER, aluminum-adsorbed vaccine containing killed whole culture of E. rhusiopathiae strain Tama 96 of serotype 2), "Nisseiken" ARBP/Swine erysipelas combined inactivated vaccine (BPSER, formulated with the killed cells of Bordetella bronchiseptica, E. rhusiopathiae strain Tama 96, and Pasteurella multocida toxoid), and "Nisseiken" Swine erysipelas live vaccine C (SEL). Reconstituted live vaccine contained approximately $10^{8} \mathrm{CFU}$ of E. rhusiopathiae strain Koganei 65-0.15 (serotype 1a) per ml.

Immunization and challenge experiments

A total of 250 female mice were used. Each of 50 mice was immunized subcutaneously twice at 2-week intervals with $0.5 \mathrm{ml}$ of SER or BPSER or one time with $0.1 \mathrm{ml}$ of SEL. The remaining 100 mice served as non-immunized controls. At 10 days after injection of SEL and 14 days after the second injection of SER or BPSER, the control and immunized mice were subdivided into groups of 10 each, and each group was challenged subcutaneously with $3.0 \times 10^{2}$ CFU of strain Fujisawa, $3.9 \times 10^{2}$ CFU of Kumamoto-S1, $3.6 \times 10^{2} \mathrm{CFU}$ of Gunma-657, $3.5 \times 10^{2}$ of Gunma-649, or $3.2 \times 10^{2} \mathrm{CFU}$ of Nagano-16. Mouse mortality was monitored daily for the following 10 days.

Ten male crossbred (Landrace $\times$ White Yorkshire $\times$ Duroc) pigs at the age of 4 weeks obtained from a conventional farm free from porcine reproductive and respiratory syndrome (PRRS) and Actinobacillus pleuropneumoniae (App) infections were used. All piglets at the farm were immunized with porcine circovirus type 2 vaccine (Ingelvac CircoFLEX). Anti-PRRS and -App antibodies were detected by using Enzyme-Linked Immunosorbent Assay (ELISA) as described previously (Metiner and Ak 2007). For experiment with vaccine SER, six pigs were randomly divided into two groups of 3 pigs each. One group was immunized intramuscularly with two doses of $1 \mathrm{ml}$ of vaccine SER at weeks 0 and 3. The second group served as non-immunized control. At week 5, the pigs of both groups were challenged intradermally with $1.0 \times 10^{7} \mathrm{CFU}$ of E. rhusiopathiae strain Gunma-649. After the challenge, clinical signs of erysipelas, including increased body temperature, depression of activity, skin lesions, and death, were monitored and recorded. Pigs were euthanized by intravascular administration of pentobarbital sodium, and autopsied at week 6. Pig hearts, lungs, livers, spleens, kidneys, lymph nodes, tonsils, synovial fluid of hock joints, and skin erythema lesions were used for bacterial isolation. Blood samples taken from the jugular vein at weeks 0 (before vaccination), 3 (before booster), 5 (before challenge), and 6 (before autopsy) were used for determination of anti-SpaA antibodies by ELISA.

For experiment with trivalent inactivated vaccine BPSER, the remaining 4 pigs were immunized with two doses of BPSER at weeks 0 and 3 with $1 \mathrm{ml}$ each, and then challenged with $1.0 \times 10^{7} \mathrm{CFU}$ of strain Gunma-657 at week 5 . After the challenge, the pigs were handled as mentioned above.

The animals used in the present study were cared for in accordance with the guidelines for animal treatment of 
the Nippon Institute for Biological Science, which conform to the standard principles of laboratory animal care (the number of approval: 11 Research-0022A).

Indirect ELISA

The procedures used to detect SpaA-specific antibody in serum by ELISA were similar to those described previously (Imada et al. 2003). The recombinant SpaA protein containing 626 amino acids originated from spaA gene of Fujisawa strain was used as the ELISA antigen. The cut-off value of the SpaA ELISA antibody in pigs was defined as 0.1 on the basis of the mean plus 3 standard deviations (SDs) for a total of 83 serum samples collected from 63 pigs on the day of immunization and 20 control pigs on the day of challenge (data not shown). Our previous investigations showed that pigs showing a SpaA E value of 0.1 or above were resistant to challenge, and those showing a SpaA E value below 0.1 were susceptible to challenge (data not shown).

\section{Results}

After challenge exposure, all 10 mice in the control groups showed clinical symptoms such as depression and anorexia and died within 3-6 days, whereas all 10 mice in experimental groups immunized with vaccines survived without clinical signs until day 10 when the study was terminated. The mice immunized with commercial vaccines were protected against challenge with not only the virulent Fujisawa strain but also the field strains of groups 1 (Kumamoto-S1) and 2 (Gunma- 657, Gunma-649 and Nagano-16).

Table 2. Protection of pigs against challenge with Gunma-649 and Gunma-657 strains

\begin{tabular}{|c|c|c|c|c|c|c|}
\hline \multirow{2}{*}{ Immunization } & \multirow{2}{*}{ Pig no. } & \multirow{2}{*}{ Challenge strain } & \multicolumn{4}{|c|}{ Presence of clinical signs after challenge } \\
\hline & & & Temperature & Erythema & Depression & Death \\
\hline \multirow{4}{*}{$\begin{array}{l}\text { Vaccination } \\
\text { (SER) }\end{array}$} & 1 & & - & - & No & - \\
\hline & 2 & Gunma-649 & - & - & No & - \\
\hline & 3 & & - & - & No & - \\
\hline & 4 & & - & - & No & - \\
\hline \multirow[t]{3}{*}{ Control } & 5 & Gunma-649 & ++ & +++ & Yes & - \\
\hline & 6 & & ++ & +++ & No & - \\
\hline & 7 & & - & - & No & - \\
\hline \multirow{3}{*}{$\begin{array}{l}\text { Vaccination } \\
\text { (BPSER) }\end{array}$} & 8 & Gunma-657 & +++ & +++ & Yes & - \\
\hline & 9 & & - & - & No & - \\
\hline & 10 & & - & - & No & - \\
\hline
\end{tabular}

Maximum measured rectal temperature:,$-<40{ }^{\circ} \mathrm{C} ;+,>40$ to $41^{\circ} \mathrm{C} ;++, 41$ to $42^{\circ} \mathrm{C},+++,>42^{\circ} \mathrm{C}$

Skin lesion: + , urticarial lesion at the injection site; +++ , systemic erythema.

After the challenge exposure with strain Gunma-649 (group 2), 2 of 3 control pigs showed typical clinical responses to E. rhusiopathiae infection, i.e., depression, anorexia, pyrexia (above $40.5^{\circ} \mathrm{C}$ ), and generalized skin erythema (Table 2). One control pig and three SER-vaccinated pigs did not show any clinical symptoms and remained healthy for 7 days after challenge with strain Gunma-649. In another group of 4 BPSER-vaccinated pigs, one pig developed typical clinical symptoms of erysipelas infection, whereas the others were healthy for 7 days after challenge with strain Gunma-657.

Eight of 10 samples collected before vaccination had the values of SpaA-specific antibodies ranging from 0.1 to 0.61 . The values of SpaA-specific antibodies in serum samples from pigs containing the maternal antibody decreased after the first vaccination, and increased slowly before challenge. At 7 days after challenge exposure, all serum samples from immunized and control pigs had the values of SpaA antibody in the ranges of 0.55 to 2.22 , and of 0.37 to 1.62 , respectively (Table 3 ). Control pig no. 4 in the experiment with SER vaccine did not show any clinical symptoms and remained healthy for 7 days 
Table 3. ELISA detection of SpaA-specific antibodies in the sera of vaccinated or nonvaccinated pigs

\begin{tabular}{ccccccc}
\hline \multirow{2}{*}{ Immunization } & Pig no. & \multirow{2}{*}{ Challenge strain } & \multicolumn{4}{c}{ ELISA values of SpaA antibodies at week no. } \\
\cline { 4 - 7 } & & & 0 & 3 & 5 & 6 \\
\hline Vaccination & 1 & & 0.07 & $0.17(+)$ & $0.27(+)$ & $1.34(+)$ \\
$($ SER) & 2 & Gunma-649 & $0.13(+)$ & 0.09 & $0.14(+)$ & $0.56(+)$ \\
& 3 & & $0.61(+)$ & $1.33(+)$ & $1.52(+)$ & $1.75(+)$ \\
\cline { 2 - 7 } Control & 4 & & $0.43(+)$ & $0.21(+)$ & $0.16(+)$ & $0.37(+)$ \\
& 5 & Gunma-649 & $0.18(+)$ & 0.08 & 0.05 & $1.62(+)$ \\
Vaccination & 6 & & 0.07 & 0.08 & 0.03 & $1.27(+)$ \\
(BPSER) & 7 & & $0.14(+)$ & 0.07 & $0.17(+)$ & $0.79(+)$ \\
& 9 & Gunma-657 & $0.23(+)$ & $0.12(+)$ & 0.07 & $2.22(+)$ \\
& 10 & & $0.10(+)$ & 0.07 & $0.19(+)$ & $1.05(+)$ \\
& & & $0.16(+)$ & 0.05 & $0.22(+)$ & $0.55(+)$ \\
\hline
\end{tabular}

after challenge with Gunma-649 strain because it still had a high level of maternal antibody at the time of challenge (with SpaA ELISA values of 0.16). Also in the experiment with SER vaccine, abnormally high levels of SpaA antibodies in serum samples collected from the immunized pig no. 3 were unexpected.

\section{Discussion}

The majority of strains isolated from recent erysipelas outbreaks among vaccinated and non-vaccinated swine belonged to group 2 (serotype 1a) having Asp-195 and Met-203 in SpaA protein (To et al. 2012). In this study, we found that the commercial vaccines could protect mice and pigs against challenge with the recent field strains of groups 1 (Kumamoto-S1) and 2 (Gunma-657, Gunma- 649 and Nagano-16).

Earlier investigations (Wood et al. 1981) reported that mice and swine immunized with standard adsorbate erysipelas bacterins of serotype 2 strains survived after challenge with strains of serotypes 1, and 2. Later, Sawada and Takahashi (1987) found that mice and swine immunized with the live vaccine survived after challenge exposure with virulent strains of various serotypes. More recently, Ingebritson et al. (2010) also showed that mice immunized with bacterin prepared from serotype 2 were protected against challenge with the SpaA-type strains. In the present study, mice immunized with the commercial live or inactivated vaccines were completely protected against challenge with Fujisawa, and $E$. rhusiopathiae strains from recent SE outbreaks.

All pigs immunized with SER and all but one pig immunized with BPSER were protected against challenge with strains Gunma-649 and Gunma-657, respectively. Failure of protection in 1 of 4 pigs immunized with BPSER might be due to a vaccine failure. Multivalent and bivalent vaccines were shown to elicit lower antibody responses than single-antigen erysipelas vaccines (Opriessnig et al. 2004). Additionally, high levels of maternal antibodies at the time of vaccination may adversely affect the immune response induced by vaccine (Imada et al. 2003). In previous investigations, we found that high levels of maternal antibodies in pigs suppressed the antibody response to immunization with live and inactivated erypelas vaccines (data not shown). Our observation that currently available vaccines formulated with strains of serotypes 1 or 2 could induce protection in mice and swine against challenge with the recent field strains of serotype 1a is consistent with data from previous reports (Wood et al. 1981; Sawada and Takahashi 1987) that suggested that bacterins from strains of serotype 2 or live vaccines from strains of serotype 1 (SpaA-type strains) can cross-protect 
pigs against challenge with the most frequently isolated serotypes 1 and 2 (SpaA-type strains).

Erysipelas outbreaks among vaccinated swine were also reported in New South Wales, Australia (Eamens et al. 2006) and the Midwestern United States (Ingebritson et al. 2010). The failures in Australia involved multivalent and bivalent inactivated vaccines, and those in the United States were attributed to inappropriate vaccine management. The present findings could not provide satisfactory explanation for the reemergence of erysipelas outbreaks among vaccinated swine in the years 2008-2011 in Japan. Since 1992 the live vaccine for erysipelas was combined with the live vaccine for swine fever. In 2000 the cessation of vaccination for swine fever together with the higher cost of inactivated vaccines resulted in a decrease in the vaccination rate. Low vaccination rates in pig populations could be considered as a cause of SE outbreaks in non-vaccinated herds. Errors in vaccine management, administration, and timing should be considered as the potential causes of some vaccine failures (Opriessnig et al. 2004; Ingebritson et al. 2010). For examples, (i) two doses of the killed bacterins are recommended for protective immunity, and sometimes producers give only one dose; (ii) presence of high level of maternal antibody or infection with porcine reproductive and respiratory syndrome virus, and/or porcine circovirus type 2 may dampen the response to active immunity (Sakano et al. 1997; Imada et al. 2003; Opriessnig et al. 2004); (iii) the practice of mixing two or more vaccine products just before using has also been a concern as it may result in lower antibody response to each antigen; and improper vaccination programs for sows and piglets could also cause some vaccine failures. Monitoring the immune status of a herd, maternal antibody decay, vaccine immune response, and implementing a proper vaccination program with erysipelas vaccines can play an important role in the control of the disease. The present results indicate that commercially available vaccines could protect swine against challenge with SpaA-type strains isolated from recent SE outbreaks.

\section{Acknowledgements}

The authors would like to thank Dr. Osamu Taira for his help in detecting PRRS antibodies.

\section{References}

Eamens GJ, Forbes WA, Djordjevic SP 2006: Characterization of Erysipelothrix rhusiopathiae isolates from pigs associated with vaccine breakdowns. Vet Microbiol 115: 329-338

Imada Y, Goji N, Ishikawa H, Kishima M, Sekizaki T 1999: Truncated surface protective antigen (SpaA) of Erysipelothrix rhusiopathiae serotype 1a elicits protection against challenge with serotype 1a and $2 \mathrm{~b}$ in pigs. Infect Immun 67: 4376-4382

Imada Y, Mori Y, Daizoh M, Kudoh K, Sakano T 2003: Enzyme-linked immunosorbent assay employing a recombinant antigen for detection of protective antibody against swine erysipelas. J Clin Microbiol 41: 5015-5021

Ingebritson A, Roth JA, Hauer PJ 2010: Erysipelothrix rhusiopathiae: association of Spa-type with serotype and role in protective immunity. Vaccine 28: 2490-2496

Metiner K, Ak S 2007: Presence and seroprevalence of Actinobacillus pleuropneumoniae in pigs in Turkey. Acta Vet Brno 76: 237-244

Nagai S, To H, Kanda A 2008: Differentiation of Erysipelothrix rhusiopathiae strains by nucleotide sequence analysis of a hypervariable region in the spaA gene: discrimination of a live vaccine strain from field isolates. J Vet Diagn Invest 20: 336-342

Opriessnig T, Hoffman LJ, Harris, DL, Gaul SB, Halbur PG 2004: Erysipelothrix rhusiopathiae: genetic characterization of Midwest US isolates and live commercial vaccines using pulsed-field gel electrophoresis. J Vet Diagn Invest 16: 101-107

Sakano T, Shibata I, Namimatsu T, Mori M, Ono M, Uruno K, Osumi T 1997: Effect of attenuated Erysipelothrix rhusiopathiae vaccine in pigs infected with porcine reproductive respiratory syndrome virus. J Vet Med Sci 59: $977-981$

Sawada T, Takahashi T 1987: Cross protection of mice and swine given live-organism vaccine against challenge exposure with strains of Erysipelothrix rhusiopathiae representing ten serovars. Am J Vet Res 48: 81-84

To H, Sato H, Tazumi A, Tsutsumi N, Nagai S, Iwata A, Nagano T 2012: Characterization of Erysipelothrix rhusiopathiae strains isolated from recent swine erysipelas outbreaks in Japan. J Vet Med Sci 74: 949-953 
To H, Someno S, Nagai S, Koyama T, Nagano T 2010: Immunization with a truncated recombinant protein SpaC of Erysipelothrix rhusiopathiae strain 715 (serovar 18) confers protective immunity against challenge with various serovars. Clin Vaccine Immunol 17: 1991-1997

Wood RL, Booth GD, Cutlip RC 1981: Susceptibility of vaccinated swine and mice to generalized infection with specific serotypes of Erysipelothrix rhusiopathiae. Am J Vet Res 42: 608-614 